\title{
La stèle de Silicia Namgidde : une épitaphe africaine (CIL XIII, 3147)
}

The stele of Silicia Namgidde: an African epitaphe (CIL XIII, 3147)

\section{Nicolas Katarzynski}

\section{(2) OpenEdition}

\section{Journals}

Édition électronique

URL : http://journals.openedition.org/abpo/3277

DOI : $10.4000 /$ abpo.3277

ISBN : 978-2-7535-5185-5

ISSN : 2108-6443

Éditeur

Presses universitaires de Rennes

Édition imprimée

Date de publication : 28 juillet 2016

Pagination : 7-25

ISBN : 978-2-7535-5183-1

ISSN : 0399-0826

\section{Référence électronique}

Nicolas Katarzynski, «La stèle de Silicia Namgidde : une épitaphe africaine (CIL XIII, 3147) », Annales de Bretagne et des Pays de l'Ouest [En ligne], 123-2 | 2016, mis en ligne le 28 juillet 2018, consulté le 02 mai 2019. URL : http://journals.openedition.org/abpo/3277 ; DOI : 10.4000/abpo.3277 


\title{
La stèle de Silicia Namgidde : une épitaphe africaine (CIL XIII, 3147)
}

\author{
Nicolas KATARZYNSKI \\ Post-doctorant à l'université Lumière Lyon II, Laboratoire HISoMA, UMR 5189
}

Lorsqu'un touriste visite Corseul (Côtes-d'Armor), les vestiges archéologiques qu'il peut admirer sont le temple de Mars, le jardin des antiques et la "stèle " de Silicia Namgidde dans l'église communale. Celle-ci relate le décès de cette femme qui avait suivi son fils à Corseul; ce dernier a élevé un tombeau en sa mémoire. L'inscription est l'une des rares et des mieux conservées de la ciuitas des Coriosolites. La mention de l'origine étrangère sur une inscription à Corseul a suscité plusieurs publications sans paradoxalement pousser à une étude plus approfondie du support ainsi que de l'épitaphe ${ }^{1}$. Notre intention est donc de mener une étude globale sur l'inscription coriosolite.

C'est en extrayant des matériaux destinés aux constructions de SaintMalo que des vestiges antiques ont été découverts par hasard à Corseul en $1709^{2}$. Un ingénieur de Saint-Malo a été envoyé sur les lieux afin d'étudier ces vestiges. Siméon de Garangeau en a rapporté une relative bonne lecture qui a été publiée par l'Académie des inscriptions et belles-lettres en $1717^{3}$. Au XVIII siècle, la pierre se trouvait scellée sur un " pilier octogone "

1. SEYMOUR DE RICCI, Robert, a été parmi les premiers commentateurs de l'inscription dans : "Répertoire épigraphique de la Bretagne occidentale ", Mémoires de la Société d'émulation des Côtes-du-Nord, 35, 1897, p. 262-263. Il a donné une bibliographie se référant à un moment ou un autre à l'épitaphe, p. 263-264. Guy GuEnNou a donné un premier commentaire aussi complet que possible dans : La cité des Coriosolites, Diplôme d'Études Supérieures, Dossiers du CeRAA, 1981, p. 83-84. MATHIEU Nicolas parle de l'inscription pour signaler une mobilité des étrangers à Corseul, avec HENRY, Paul, "Corseul : Lever de rideau sur une capitale? ", Annales de Bretagne et des Pays de l'Ouest, 2003, p. 20. Les autres, à l'image de LANGOUET, Loïc, Les Coriosolites. Un peuple armoricain, de la période gauloise à l'époque gallo-romaine, Dossiers du CeRAA, Saint-Malo, 1988, ont été plus discrets.

2. De Saint-Jouan, Régis, Département des Côtes-d'Armor. Dictionnaire des communes, éléments d'histoire et d'archéologie, Conseil général des Côtes-d'Armor, Saint-Brieuc, 1990, p. 170.

3. ANONYME, « De l'ancienne ville des Curiosolites », dans L'Histoire de l'Académie royale des inscriptions et belles-lettres, I, Paris, 1717, p. 294-298: « Mais ce qui est de plus remar- 
de l'église Saint-Pierre dont la première mention remonte à $1123^{4}$. Le bâtiment remontait au Xve siècle au moins d'après la datation d'un porche de la même époque qui fut réemployé plus $\operatorname{tard}^{5}$. Un fragment a également été conservé dans la tour de cette église selon Nadaud ${ }^{6}$. Toutefois, son existence est douteuse ${ }^{7}$. Une " colonne avec sa base " dans le mur du cimetière est signalée en $1820^{8}$. Enfin, sur la place de l'église, sept monnaies dont une de Constance et une de Victorinus ont été mises au jour ${ }^{9}$. Au moment de la reconstruction de l'église en 1836, la pierre a été remployée dans le mur sud, à l'angle du transept. L'inscription a été publiée au XIX ${ }^{\mathrm{e}}$ siècle dans le Corpus Inscriptionum Latinarum sous le XIII, numéro 3147. R. Seymour de Ricci indique que le moulage se trouve " au musée de Dinan ${ }^{10}$ ". Elle est classée comme " objet d'art ", monument historique, le $1^{\mathrm{er}}$ mai $1911^{11}$.

L'inscription a sans doute été découverte ailleurs et déplacée depuis un lieu indéterminé situé près de l'agglomération antique. Selon la $C A G$ (Carte Archéologique de la Gaule), plusieurs auteurs des XVIII ${ }^{\mathrm{e}}$ et XIX ${ }^{\mathrm{e}}$ siècles ont pensé au Courtil Saint-Antoine, à l'est du bourg. Mais cette nécropole serait tardive alors que l'inscription date du II $^{\mathrm{e}}$ siècle (nous reviendrons sur la datation plus loin). D'autres lieux sont possibles. Le site du Champ Mulon a livré deux inscriptions dans les parcelles 359, 360 et 383 de la section M du cadastre dit napoléonien ${ }^{12}$. Selon la $C A G$, ces inscriptions pouvaient

quable, est une grande pierre de cinq pieds de long, large \& épaisse de trois, que l'on a tirée d'un tombeau, pour en faire un pilier octogone, auquel on a laissé une face plus large que celles qui luy répondent, pour conserver une inscription Latine, telle qu'elle est figurée dans la copie suivante. D * M. * S SILICIA. NA./ MGIDDE-DO/MO. AFFRIKA/EXIMIA PIETATE/FILIVM SECVTA/HIC. SITA. EST/VIXIT. AN - LXV/CN. IANVARI/VS FIL-POSVIT ", p. 295. Pour d'autres détails sur la découverte des vestiges archéologiques à Corseul à la même époque, voir BESNIER, Maurice, "Note sur un plan des ruines de Corseul (Côtesdu-Nord), daté de 1709 ", Bulletin et mémoires de la Société nationale des antiquaires de France, 8, Paris, 1909, p. 82-96.

4. Couffon, René, "Répertoire des églises et chapelles du diocèse de Saint Brieuc et Tréguier ", Société d'émulation des Côtes-du-Nord, LXX, 1939, p. 93.

5. De Saint-Jouan, Régis, Département des Côtes-d'Armor..., op. cit., p. 169; Couffon, René, op. cit., p. 93.

6. CIL XIII, 3146. Nadaud a lu aedificauit bien que l'inscription soit illisible de son aveu, cf. du même auteur, "Lettres sur Dinan, Corseul, Saint-Malo, Dol, le Mont Saint-Michel, etc. quatrième lettre. Corseul ", Le Lycée armoricain, 1, 1823, p. 405. D'ailleurs, le verbe n'est mentionné que dans les inscriptions chrétiennes en Lyonnaise et en Aquitaine.

7. Guennou, Guy, La cité des Coriosolites, op. cit., p. 83; Kerebel, Hervé, Le Roux, Patrick, " Une dédicace fragmentaire découverte en 1991 à Corseul (Côtes-d'Armor) ", Revue archéologique de l'Ouest, 11, 1994, p. 165.

8. GuEnnou, Guy, La cité des Coriosolites, op. cit., p. 21.

9. Ibidem, p. 22.

10. Seymour DE Ricci, Robert, " Répertoire épigraphique de la Bretagne occidentale ", art. cit., p. 262.

11. De Saint-Jouan, Régis, Département des Côtes-d'Armor..., op. cit., p. 170.

12. CIL XIII, 3144 et 3145. Guennou, Guy, La cité des Coriosolites, op. cit., p. 18-19, 83; KEREBEL, Hervé, LE RouX, Patrick, « Une dédicace fragmentaire découverte en 1991 à Corseul (Côtes-d'Armor) ", art. cit., p. 165-166; Bizien-Jaglin, Catherine, Galliou, Patrick, KEREBel, Hervé, Carte archéologique de la Gaule : les Côtes-d'Armor, Académie des Inscriptions et Belles-Lettres, Paris, 2003, p. 121 (désormais abrégé $C A G$ suivi du numéro du dépar- 
venir d'une nécropole implantée juste à l'ouest du cimetière moderne, à la hauteur du chemin Saint-Jean. Un autre fragment a été découvert au Clos Julio, à l'est de l'église ${ }^{13}$. Il proviendrait d'une nécropole au sud-est de l'agglomération antique.

\section{Le support}

\section{Dimensions}

150 (H) x 58 (L) x 17 (ép.) cm. Lettres : 13 cm (1. 1), 9 cm (1. 2), 7 cm (1. 3), 6,5 cm (1. 4-8), 5,5 cm (1. 9-10).

L'inscription (fig. 1) est gravée dans un bloc de granit comme deux autres inscriptions (CIL XIII, 3143; ILTG, 340). Ce granite est issu du massif de Dinan qui a souvent fourni les meilleurs granites. Il est extrait dans les carrières de Brusvily, du Hinglé et de Languédias. La majorité des blocs de grand appareil d'origine antique sont en granite de Languédias issu de la carrière qui se situe au sud de Corseul ${ }^{14}$. Le monument est probablement taillé dans le granite de Languédias qui est la matière préférée des bâtisseurs romains ${ }^{15}$ qui n'ont pas hésité à faire venir ce matériau pour tailler des bases à Rennes ${ }^{16}$. L'inscription doit son bon état à sa conservation sous les toits de l'église pendant 200 ans $^{17}$. Le bord gauche a été retaillé comme en témoigne le $S$ à moitié coupé à la ligne 2. Cela atteste un remploi de la pierre antérieur à la visite de l'ingénieur malouin. F. Rever, qui a vu sur place le monument en 1823 , confirme le fait ${ }^{18}$. Si le côté gauche est manifestement

tement puis du numéro de page). Sur le cadastre, voir Archives départementales des Côtes-d'Armor, 3 P 53/1, consultable sur le site internet des Archives départementales.

13. ILTG, 340; KEREBEL, Hervé, LE Roux, Patrick « Une dédicace fragmentaire découverte en 1991 à Corseul (Côtes-d'Armor)", art. cit.; CAG/22, ibidem.

14. ÉVEILLARD, Jean-Yves, "Les types de pierre de construction en Armorique romaine ", dans ÉVEILLARD, Jean-Yves et al., La pierre de construction en Armorique romaine. L'exemple de Carhaix, Centre de Recherche Bretonne et Celtique (CRBC), 1998, p. 18-19.

15. ÉVEILLARD, Jean-Yves, "Les types de pierre de construction en Armorique romaine ", art. cit., p. 19. Pour GALLIOU, Patrick, Les tombes romaines d'Armorique. Essai de sociologie et d'économie de la mort, Documents d'Archéologie Française (DAF), Maison des Sciences de l'Homme, Paris, 1989, p. 101, il s'agit du " granit de Kérinan ». Ce nom est le lieu-dit de Mégrit, commune située tout près de Languédias. Voir les remarques d'ÉVEILLARD, JeanYves, "La sculpture sur pierre de l'époque romaine et de l'âge du Fer en Bretagne : des collections embryonnaires avant 1941 ", Annales de Bretagne et des Pays de l'Ouest, 118/3, 2011, p. 106, sur les matériaux utilisés pour la sculpture (granite local meilleur marché, calcaire importé pour des œuvres ou des monuments plus prestigieux).

16. ÉVEILLARD, Jean-Yves, "Les types de pierre de construction en Armorique romaine ", art. cit., p. $21 ; A E 1969-70,405 a$.

17. On voit une différence de conservation entre cette pierre et certains monuments de Nantes où certaines inscriptions sont presque effacées. Nous le constatons sur la photo de l'inscription $n^{\circ} 49$ a de Gallou, Patrick, Les tombes romaines d'Armorique..., op. cit., p. 140 (CIL XIII, 3114), no 49b (CIL XIII, 3117), no 49c (CIL XIII, 3119). D'autres sont mieux conservées telle l'ins. no $50 \mathrm{~b}$ (CIL XIII, 3126) mais restent exceptionnelles.

18. REVER, François, «Sur Corseul. A M. Ed. Richer », Le lycée armoricain, 2, 1823, p. 273. Voir plus bas pour l'analyse du S. 
Figure 1 - Corseul, inscription de l'église Saint-Pierre (cl. N. Katarzynski)

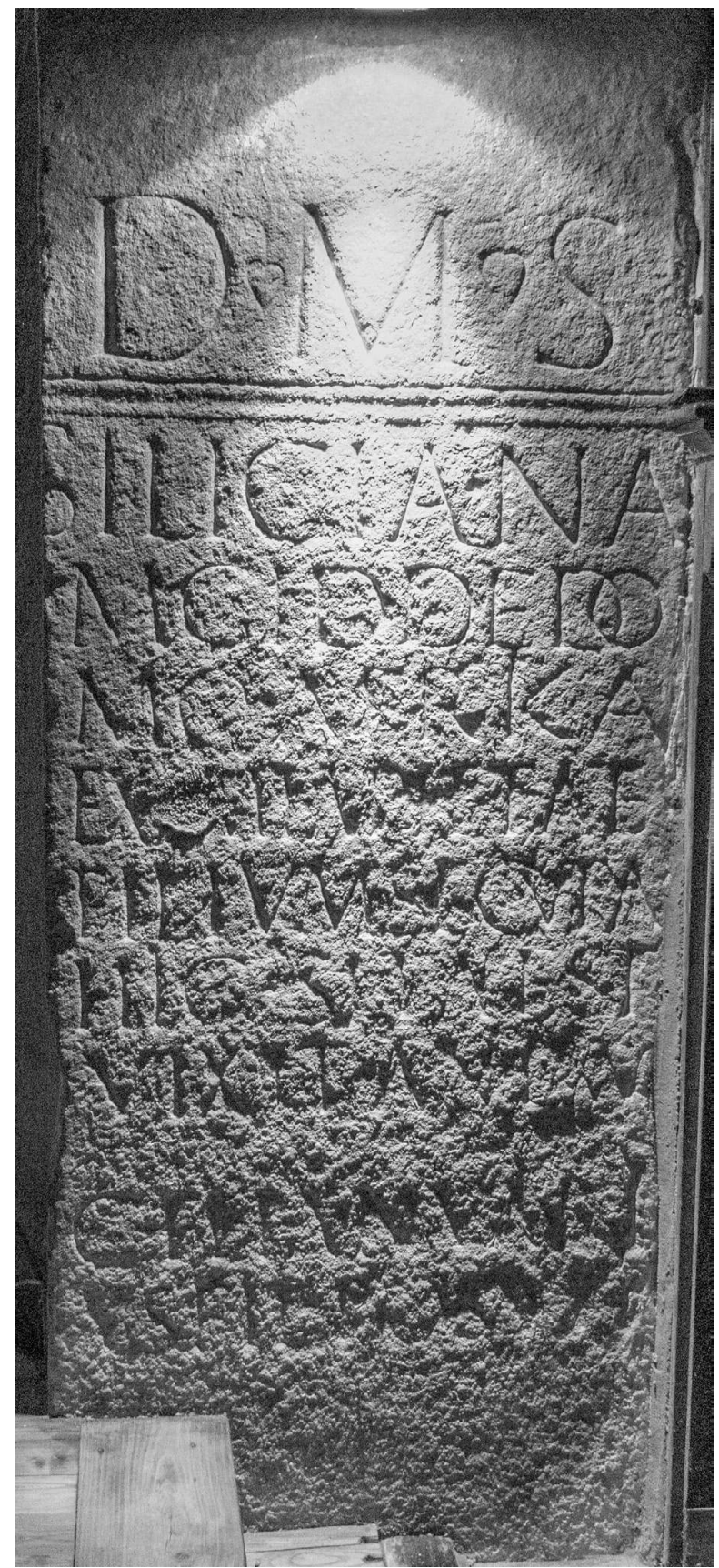


coupé, il est probable que le côté droit l'est également. Un court espace vide sur tout le bord gauche montre un souci de l'ordinatio. Or, il manque clairement un court espace vide à droite. En apparence, le texte est gravé sur toute la surface du support mais il faut remarquer une double ligne moulurée qui sépare les initiales $D$ (is) $M$ (anibus) $S$ (acrum) du reste du texte (entre la ligne 1 et les autres lignes). Cette double ligne donne l'impression que l'inscription présente deux champs épigraphiques distincts.

À cause du remploi de la pierre, la nature exacte du monument n'apparaît pas immédiatement et doit être précisée. Base, autel ou stèle, le monument ne fait pas l'unanimité parmi les historiens ${ }^{19}$. Une base est un support monumental destiné à soutenir une statue (plus rarement une colonne). Les dédicaces religieuses ou honorifiques privilégient les bases. Une base dédiée à Neptune Hippius à Douarnenez (Finistère) avec la statue du dieu ${ }^{20}$ ne déroge pas à la règle. Nous cherchons en vain des bases à vocation funéraire dans les ILN (Inscriptions Latines de Narbonnaise) d'Apt, Antibes, Riez ou encore dans l'épigraphie des Alpes-Maritimes. Elles sont rares (ILN, Aix, $41,66^{21}$ ou ILN, Riez, 43). Il est douteux de voir en l'inscription locale une base de statue. Restent donc l'autel et la stèle. La forme de l'autel consiste surtout en un dé parallépipédique ou cylindrique, dont le couronnement est formé par une corniche surmontée d'une cimaise ${ }^{22}$, reposant sur un petit soubassement ${ }^{23}$. Un autel (ou une base) peut être remployé suivant deux méthodes. Soit le couronnement et le socle sont arasés à l'aplomb du dé afin de sceller plus efficacement le monument dans le mur. Cette méthode est connue à Vence (Alpes-Maritimes) où plusieurs autels sont

19. N. Mathieu hésite entre un " cippe " et une base, cf. du même auteur, "L'épigraphie dans l'Ouest armoricain, historiographie et constitution des collections locales ", Annales de Bretagne et des Pays de l'Ouest, 118/3, 2011, p. 137. La CAG le considère comme un autel funéraire puis comme une stèle (CAG/22, p. 121). G. GUENNOU, H. KEREBEL, P. LE RouX, ainsi que L. LANGOUET, pensent à un autel, cf. des mêmes auteurs, GuENNOU, Guy, La cité des Coriosolites, op. cit., p. 83; KereBel, Hervé, LE RouX, Patrick, " Une dédicace fragmentaire découverte en 1991 à Corseul (Côtes-d'Armor) ", art. cit., p. 165; LANGOUËT, Loïc, Les Coriosolites..., op. cit., p. 31. Chez H. KerEBEL et P. Le RouX, l'inscription votive du site de Montafilant est considérée comme une stèle (CIL XIII, 3143) alors que l'épitaphe est prise pour un autel. L. LANGOUET privilégie aussi la stèle pour la même inscription. Quant à G. GuENNOU, il pense à l'autel, cf. du même auteur, 1981, p. 29, mais emploie le terme de stèle à la p. 79

20. SANQUER, René, "Une nouvelle lecture de l'inscription à Neptune trouvée à Douarnenez (Finistère) et l'industrie du garum armoricain ", Annales de la Bretagne, 80, 1973, p. 215-236; ÉvEILlaRd, Jean-Yves, "Actualité de la statue d'époque romaine en Bretagne (II) ", Aremorica, 3, 2009, p. 82 ; ÉveILlard, Jean-Yves, LE Roux, Patrick, "L'inscription de Douarnenez à Neptune Hippius : une mise à jour ", Aremorica, 6, 2013, p. 7-16.

21. On se demande parfois si ce ne sont pas des autels à cause de l'absence de description de trous de scellement de statues ou d'indices de statue dans l'ILN.

22. BonNEVILLE, Jean-Noël, " Le support monumental des inscriptions : terminologie et analyse ", dans Espagne Hispanique, problèmes de méthode et d'édition, Éd. De Boccard, 1984, p. 134.

23. Cagnat, René, Снарот, Victor, Manuel d'archéologie romaine, Picard, Paris 1916-1920, p. 138. 
scellés dans le mur extérieur de la cathédrale ${ }^{24}$. Soit le couronnement et le socle ont été ôtés du dé médian. Plusieurs bases de cette sorte ont été découvertes à Rennes ${ }^{25}$. Ce serait cette méthode qui aurait prévalu ici. Le double sillon, inséré ici entre les deux premières lignes, est cependant problématique. Si le monument était un autel, une surface de la face antérieure du couronnement aurait porté la marque d'une corniche. Ce double sillon devrait normalement être saillant. Il aurait clairement délimité l'arase et le champ épigraphique. Toute la surface de la face antérieure du monument est pourtant plate malgré le sillon. Celui-ci ne ressemble à aucune moulure parmi les exemples de la typologie établie par J.-N. Bonneville ${ }^{26}$. La taille de l'espace vide (24 cm) au-dessus des lettres DMS est plutôt exagérée pour un couronnement d'autel. La formule Dis Manibus peut se placer soit sur le couronnement, dans les acrotères ou les volutes des coussinets, soit dans le champ épigraphique situé sur le dé. Bien que nous n'ayons pas la prétention d'avoir vu toutes les photographies des autels d'Occident ${ }^{27}$, nous n'avons pas (encore) vu les initiales $D M S$ gravées sur un couronnement. Elles sont situées dans le champ épigraphique sur le dé. Contrairement à ces initiales, $D M$ est gravé plusieurs fois sur le couronnement à l'instar des inscriptions de Lyon (CIL XIII, 1849, 1856, 1858, 1865, 1868) ou de Sens (CIL XIII, 2946), sur les acrotères (ILN, Antibes, 8, 109; CIL XII, 2832) ou sur les volutes des coussinets (CIL XII, 39). L'autel est donc une option plutôt douteuse.

L'hypothèse de la stèle se renforce donc par élimination. La définition du monument a suscité une abondante discussion, en grande partie résumée par J.-N. Bonneville ${ }^{28}$. Selon nous, une stèle est un monument autonome, isolé et/ou pouvant être intégré dans une structure architecturale, dont la taille d'épaisseur ne doit pas excéder celle d'un autel ou d'une base de grande dimension ${ }^{29}$. Une stèle est un monument dont la hauteur est ordi-

24. KATARZYNSKI, Nicolas, Memorias et titulos fecerunt in Alpibus Maritimis : la construction des modèles régionaux et locaux dans l'épigraphie latine impériale des Alpes Maritimae, thèse dactylographiée, université Lumière Lyon II, Lyon, 2014, p. 90.

25. BousQuET, Jean, «Inscriptions de Rennes ", Gallia, n 29, 1971, p. 109-122.

26. Il y a en tout 35 exemples, BonNEvILLE, Jean-Noël, « Le monument épigraphique et ses moulurations ", Faventia, n 2, 1980, p. 91-98.

27. Surtout lorsqu'elles ne sont pas publiées dans les recueils d'épigraphie, les $C A G$ ou sur Internet. Les photos ne sont pas systématiquement publiées dans les $C A G$. Les Supplementa Italica mentionnent les nouvelles inscriptions ou redécouvertes qui en complètent (ou corrigent) le CIL, excluant de fait les monuments encore conservés cités dans le recueil. Le corpus des inscriptions des Trois Gaules (ILTG) ne publie pas de photographie. La publication des photos dans une banque de données sur Internet, EpigraphikDatenbank Clauss-Slaby, repose sur une bonne volonté des contributeurs.

28. Bonneville, Jean-Noël, " Le support monumental des inscriptions... ", art. cit., p. $120-123$.

29. Une taille domine le plus souvent à partir de $40 \mathrm{~cm}$ chez un autel ou une base de grande dimension, ILN, Apt, $22(60 \mathrm{~cm}), 23(46 \mathrm{~cm}), 39(54 \mathrm{~cm})$, etc. ; ILN, Aix, 8 (45-46 cm), $97(42 \mathrm{~cm}), 154(41 \mathrm{~cm})$, etc.; KATARZYNSKI, Nicolas, Memorias et titulos fecerunt in Alpibus Maritimis..., op. cit., p. 94-95, 105-106. Nous trouvons moins de $30 \mathrm{~cm}$ en général chez une stèle, ibidem, p. 41-42. 
nairement supérieure à la largeur ${ }^{30}$. La stèle doit être dressée de manière à être lue de face en prioritée ${ }^{31}$. La stèle et la plaque se distinguent par leur fonction. La plaque est pour l'essentiel une pierre destinée à être scellée dans une structure ${ }^{32}$ dont la taille de l'épaisseur du monument est inférieure à $20 \mathrm{~cm}$, plus particulièrement entre 5 et $10 \mathrm{~cm}^{33}$. Les stèles sont peu abondantes dans la ciuitas des Coriosolites ou à proximité de celle-ci. Une stèle en granit remployée se trouve à Saint-Igneuc, au sud-ouest de Corseul. Une décoration a été sculptée sur les deux faces du monument. Sur la face antérieure, on peut apercevoir deux pilastres soutenant une archivolte qui encadre un fronton et deux motifs en forme de C. Sous le fronton, se lisent les initiales $D M$ séparées par un large espace vertical ${ }^{34}$. Un tel monument avec ses deux faces lisibles n'est pas unique en Armorique. On le retrouve sur une autre stèle anépigraphe, sans doute votive, à Bécherel ${ }^{35}$. Il convient d'ajouter au dossier un fragment en granite trouvé au Clos-Julio ${ }^{36}$. Le même texte est gravé sur les faces opposées. Son épaisseur $(16 \mathrm{~cm}$ qui reste l'une des plus faibles épaisseurs. Le seul élément de comparaison est la stèle de Namgidde elle-même qui mesure $17 \mathrm{~cm}$...) est tout à fait conforme au modèle des stèles ${ }^{37}$. La lecture sur deux faces montre un monument sans

30. Les stèles en position horizontale sont exceptionnelles. Un exemplaire à Cimiez (Alpes-Maritimes) présente deux inscriptions gravées côte à côte sur une même surface horizontale, $A E 1981,603$.

31. KATARZYNSKI, Nicolas, Memorias et titulos fecerunt in Alpibus Maritimis..., op. cit., p. 26.

32. Les stèles intégrées dans une structure ne semblent pas être légion. Elles sont surtout utilisées comme des bornes de délimitation funéraire. À Narbonne, on a dégagé les fondations d'un enclos funéraire à plan quadrangulaire. Dans la façade ouest, une stèle a été retrouvée, debout et encastrée dans le mur antique. Une autre stèle présente des dimensions de 12 pieds, $C A G / 11$, p. 336. Michel Tarpin mentionne quatre stèles placées à chaque angle du monument d'Hirpius (décédé en 43 av. J.-C.) à Rome. Leur position matérialise le mur entourant le tombeau, cf. du même auteur, "À propos d'une stèle à sommet cintré du Musée de Lyon ", dans LE BoHEc, Yann (éd.), L’Afrique, la Gaule, la religion à l'époque romaine, Mélanges Marcel Le Glay, coll. "Latomus; 226 ", 1994, p. 337.

33. KATARZYNSKI, Nicolas, Memorias et titulos fecerunt in Alpibus Maritimis..., op. cit., p. 59. Les plaques chez les Voconces Septentrionaux mesurent en moyenne 7,5 cm, DESAYE, Henri, "Les épitaphes des Voconces Septentrionaux ", RAN, n 36, 2003, p. 387.

34. $C A G / 22$, p. 168-169. ÉvEILLARD, Jean-Yves, "La sculpture sur pierre de l'époque romaine et de l'âge du Fer en Bretagne... ", art. cit., 2011, p. 106, trouve une ressemblance avec la collection des stèles de Nantes par son décor architectural (pilastres, archivolte encadrant un fronton).

35. $C A G / 35$, p. 55 ; SANQUER, René, "Informations archéologiques. Circonscription de Bretagne ", Gallia, n 35/2, 1977, p. 336; ÉVEILLARD, Jean-Yves, "Sur quelques aspects originaux de la sculpture en pierre de l'Armorique romaine ", Annales de Bretagne et des Pays de l'Ouest, 105, 1998, p. 84-86; GAlliou, Patrick, op. cit., p. 97-98.

36. Merlat, Pierre, "V Ve circonscription. Côtes-du-Nord ", Gallia, 1957, p. 177-78; ILTG, 340; Galliou, Patrick, Les tombes romaines d'Armorique..., op. cit., p. 101-102.

37. Les monuments de Nantes mesurent entre 18 et $33 \mathrm{~cm}$, GALLIOU, Patrick, Les tombes romaines d'Armorique..., op. cit., p. 140-145. Il est regrettable que la taille d'épaisseur ne soit pas donnée par P. Galliou pour la plupart des stèles. Ce défaut est corrigé dans la $C A G / 44$ pour certaines stèles manquantes. La stèle de Saint-Igneuc mesure $14 \mathrm{~cm}$, cf. $C A G / 22$, p. 168. Le monument de Bécherel mesure $25 \mathrm{~cm}$ d'après la taille du mur dans laquelle est scellée la stèle, cf. SANQUER, René, "Informations archéologiques... ", art. cit., p. 336. L'épigraphie des Alpes-Maritimes présente une taille entre 13 et $40 \mathrm{~cm}$, 
doute fiché dans le sol ce qui en fait probablement une stèle. Il est trop tôt pour conclure à un trait original de la stèle à double face dans la région entre les Coriosolites et les Riedones à cause du faible nombre des occurrences $^{38}$. La stèle coriosolite a un style ornemental très dépouillé (deux hederae et un double sillon). Il a très peu à voir avec la typologie nantaise (pilastres reposant sur un socle et encadrant un décor ou une épitaphe, fronton triangulaire à décor, symboles astraux, personnages sculptés dans une niche) ${ }^{39}$ ou la stèle de Saint-Igneuc. La partie inférieure du monument semble être lisse. Une surface non lisse dans la partie inférieure signale une volonté d'enterrer partiellement une stèle. Nous le constatons clairement sur les stèles de Pessicinnus Sabinus (CIL XIII, 3114), Famulus (CIL XIII, 3117) et Priscilla (CIL XIII, 3128) à Nantes ${ }^{40}$. La partie inférieure de la stèle coriosolite pourrait avoir été découpée pour les besoins d'un remploi. Toutefois, il existe des stèles dont la partie inférieure n'est pas laissée à l'état brut ${ }^{41}$. Nous ne pouvons pas nous avancer sur ces points. Nous ignorons si la partie supérieure a été retaillée. Si tel est bien le cas, le sommet aurait pu être cintré $^{42}$, triangulaire ${ }^{43}$ ou plus simplement rectangulaire ${ }^{44}$. Enfin, la hauteur de la stèle est remarquable. Très peu de stèles de Bretagne la dépassent ${ }^{45}$. La taille pourrait avoir été plus élevée si la stèle a été retaillée.

\title{
Transcription, développement et traduction de l'inscription
}

\author{
$D \bullet M \bullet S$ \\ SILICIA • NA \\ MGIDDE DO \\ $M O \bullet A F R I K A$ \\ EX[.]MIA PIETATE
}

KataRZYNSKI, Nicolas, Memorias et titulos fecerunt in Alpibus Maritimis..., op. cit., p. 40-42. La taille moyenne des lettres du fragment est $8 \mathrm{~cm}$ (MERLAT, Pierre, "Ve circonscription. Côtes-du-Nord ", art. cit., p. 177, n 17), un chiffre comparable à $9 \mathrm{~cm}$ de la deuxième ligne de notre inscription.

38. D'autant plus que le décor architectural de Saint-Igneuc est plus dans le registre nantais (autel, pilastres, sommet triangulaire), cf. ÉvEILLARD, Jean-Yves, " La sculpture sur pierre de l'époque romaine et de l'âge du Fer en Bretagne ", art. cit., 2011, p. 106.

39. Galliou, Patrick, Les tombes romaines d'Armorique..., op. cit., p. 76, 140-145 (avec photographies).

40. Ibidem, p. 140, fig. 49a, b; p. 142, fig. 50d. Photographie de la stèle de Regalus, MathIEU, Nicolas, "L'épigraphie dans l'Ouest armoricain... ", art. cit., p. 28, fig. 4.

41. Notamment le CIL XIII, 3126 (fig. 50b) ou les monuments anépigraphes à la p. 144 de Gallou, Patrick, Les tombes romaines d'Armorique..., op. cit.

42. Deux exemples à Bécherel et Nantes (CIL XIII, 3114; Galliou, Patrick, Les tombes romaines d'Armorique..., op. cit., p. 76, 140, fig. 49a, et 141).

43. Quatre stèles à Nantes (CIL XIII, 3119, 3124; GALLIOU, Patrick, Les tombes romaines d'Armorique..., op. cit., p. 143, 145).

44. Trois stèles à Nantes, ibidem, p. 76, 142 (fig. 50b), 144 (fig. 51a-b).

45. CIL XIII, $3126(155 \mathrm{~cm}), 3129$ (169 cm); GALLIOU, Patrick, op. cit., p. 143, no $19(150 \mathrm{~cm})$, ibidem, p. $145, \mathrm{n}^{\circ} 22(175 \mathrm{~cm})$. 
FILIVM SECVTA

HIC SITA EST

VIXIT AN LXV

CFL IANVARI

VS FIL POSVIT

$D$ (is) M(anibus) S(acrum).//Silicia $\mathrm{Na} /$ mgidde, do/mo Afrika,/ex[i]mia pietate/filium secuta,/hic sita est,/uixit an(nis) LXV./C(aius) Fl(auius) Ianuari/ us, fil(ius), posuit.

"Consacré aux Dieux Mânes. Silicia Namgidde, originaire d'Afrique, a suivi son fils avec une rare affection. Elle repose ici. Elle a vécu soixantecinq ans. Son fils Caius Flavius Ianuarius a élevé (le monument). »

Le champ épigraphique n'était pas mouluré. La gravure est bonne sans se rapprocher de la haute qualité de la dédicace sur plaque de marbre du site de Monterfil II ${ }^{46}$. Les lettres varient de $13 \mathrm{~cm}$ à la $1^{\text {re }}$ ligne, $9 \mathrm{~cm}$ à la 1.2 , $7 \mathrm{~cm}$ à la l. $3,6,5 \mathrm{~cm}$ sur les 1.4 à 8 et enfin $5,5 \mathrm{~cm}$ sur les dernières lignes. La hauteur des lettres diminue progressivement. Elle passe de 13 à $5,5 \mathrm{~cm}$. Ceci pourrait laisser penser que le lapicide s'est efforcé de raccourcir les lettres par manque de place. Un fait met cependant en doute cette hypothèse : il y a encore de la place en bas de l'inscription. Le graveur pouvait donc aisément terminer son travail sans recourir à ce moyen. D'autre part, il y a un petit espace vide entre la $8^{\mathrm{e}}$ et $9^{\mathrm{e}}$ ligne. Il s'agit d'un espace de séparation entre le texte centré sur la défunte et les lignes suivantes mentionnant le dédicant. Les premières lettres ont volontairement été grossies afin de mettre l'accent sur l'identité de la défunte. Notons en effet le contraste évident entre les grosses lettres du nom de la défunte et les petites lettres du dédicant. L'identité de Silicia Namgidde importe beaucoup plus dans le contexte du texte funéraire. D'après R. Seymour de Ricci, en 1897, une barre de fer a été scellée dans l'inscription " en détruisant les trois premières lettres de la cinquième ligne ${ }^{47}$ ". En réalité, seule la lettre I d'eximia a disparu ${ }^{48}$. Une grande partie de $\mathrm{M}$ a été détruite. La détérioration de ces

46. KEREBEL, Hervé, Le Roux, Patrick, " Une dédicace fragmentaire découverte en 1991 à Corseul (Côtes-d'Armor) ", art. cit., p. 165-171. Remarquer aussi la bonne gravure du fragment du Clos Julio (ILTG, 340. Photographie, GuEnNou, Guy, La cité des Coriosolites, $o p$. cit., p. 86). La qualité de la gravure de ces pièces montre une bonne expérience qui ne peut s'acquérir qu'au fil de plusieurs essais et au terme du travail assez fourni. Le nombre des inscriptions de Corseul devrait donc être plus élevé que prévu. La petite plaque de Monterfil est une pièce facilement transposable. Ceci n'interdit pas l'hypothèse d'un atelier lointain mais cela reste de la pure spéculation.

47. SEYMour DE Ricci, Robert, "Répertoire épigraphique de la Bretagne occidentale ", art. cit., p. 262.

48. La CAG/22 comme HENRY, Paul, MATHIEU, Nicolas, " Corseul : Lever de rideau sur une capitale? ", art. cit., 2003, p. 21, ont commis une erreur en mettant entre les parenthèses de restitution les lettres [EXI]. Seule la lettre I est restituable. G. GuENNOU n'a jamais publié la photographie de l'inscription dans une réédition de 1981. Il signale pourtant la disparition des lettres à cause d'une barre de fer, cf. du même auteur, p. 84. L. LANGOUET se contente de mettre les deux points avant XIMIA signalant la disparition des lettres, cf. du 
lettres existait-elle déjà au moment de la publication en 1717 ou la pierre a-t-elle subi un tel outrage au cours du temps depuis sa découverte (on pense notamment au signalement de R. Seymour de Ricci)? L'Académie des inscriptions et des belles lettres a publié en toutes lettres le mot eximia. Mais, à l'époque, les règles de publication en épigraphie latine n'existaient pas. On se souciait sans doute peu de tels détails. Nous ne pouvons pas apporter de réponse à cette question. Le premier $\mathrm{S}$ a été brisé (1. 2). Il est douteux que le graveur ait fini la lettre dans un petit espace encore vide après s'être aperçu de son oubli. Tout au long du travail, le lapicide a fait trois ligatures. Il démontre ainsi son souci de l'ordinatio. Le $S$ est le résultat du début hésitant du travail. Par la suite, le lapicide maîtrise très bien son sujet. La lettre a certainement été brisée par le remploi de la pierre. Graphie AFRIKA pour AFRICA. Elle se rencontre sur une inscription de Bulla Regia ${ }^{49}$. Ligatures $D O$ (1. 3), IK (1. 4), TE (1. 5). Petit $V$ inséré entre les lettres $C$ - $T$ de secuta (1. 6). $A$ barrés, sauf à partir de la $5^{\mathrm{e}}$ ligne (mais la détérioration de la pierre rend leur lecture difficile). Grands I dans les termes hic et Ianuari (à la fin). Deux hederae ont été gravées entre les sigles DMS. Point de séparation circulaire (1. 2). Il est le seul point à être clairement signalé sur la pierre. L'autre point, à la l. 4, n'est peut-être pas assuré. Si d'autres points sont avancés par le $C I L$, ils sont difficiles à lire.

Apparue en Narbonnaise occidentale dans la première moitié du $\mathrm{I}^{\mathrm{er}}$ siècle apr. J.-C., la formule initiale DM est employée à Vienne, Lyon et sur le Rhin à la fin du même siècle ${ }^{50}$. En Afrique, si la consécration est relativement précoce à Carthage et Dougga (début du II $^{\mathrm{e}}$ siècle), elle est plus tardive à l'ouest de la province africaine (ainsi, elle est attestée à partir d'Hadrien pour la cité de Cirta) ${ }^{51}$. Le champ lexical des sentiments ou épithètes (pientissimus, carissimus, etc.), absent ici, apparaît au cours du II ${ }^{\mathrm{e}}$ siècle avant de devenir fréquent ${ }^{52}$. Les tria nomina sont aussi un indice de datation si l'on

même auteur, p. 31. Jusqu'en 1965 ou 1981, la barre de fer est-elle restée scellée sur le monument? Je l'ignore.

49. AE 1971, 490 : Q(uinto) Aradio Rufino/Optato Aeliano co(n)s(uli)/sodal[i] Augustali agenti/uice p[r]oco(n)s(ulis) prou(inciae) Afrik(ae)/leg(ato) Au[g(usti)] pr(o) pr(aetore) prouinciarum/[Syriae] Coelae item Phoe/[nic(es) ite]m Galatiae praef(ecto)/[aer(ari) Satu]rni praef(ecto) aera/[ri mil(itaris) l]eg(ato) leg(ionis) VII[-].

50. KATARZYNSKI, Nicolas, Memorias et titulos fecerunt in Alpibus Maritimis..., op. cit., p. 187188; PIETRI, Charles, "Inscriptions funéraires latines ", dans Christiana respublica. Éléments d'une enquête sur le christianisme antique, Publications de l'École Française de Rome, 234, 1997, p. 1413. Pour Vienne, Burnand, Yves, "Chronologie des épitaphes romaines de Vienne (Isère) ", Revue des Études Anciennes, nº 63, 1961, p. 304 sq.; ILN, Vienne, I, 53; pour Lyon Audin, Amable, BuRnAnd, Yves, "Chronologie des épitaphes romaines de Lyon ", Revue des Études Anciennes, n 61, 1959, p. 322-323; pour le Rhin, WEYNAND, Rudolf, " Form und Dekoration der römischen Grabsteine der Rheinlands in erstenJahrhundert ", $B J$, CVIII-CIX, 1902 1902, p. 185-238.

51. LASSERE, Jean-Marie, "Recherche sur la chronologie des épitaphes païennes de l'Africa ", Antiquités Africaines, nº 7, 1973, p. 123-126.

52. À Vienne, les épithètes deviennent courantes à partir de la $3^{\mathrm{e}}$ époque (du $2^{\mathrm{e}}$ quart du I $^{\mathrm{e}}$ siècle au début du $\mathrm{III}^{\mathrm{e}}$ siècle), BuRnand, Yves, "Chronologie des épitaphes romaines de Vienne (Isère) ", Revue des Études Anciennes, n 63, 1961, p. 310. Dans cette cité et 
considère que les duo nomina seconde manière sont utilisés tardivement à partir de la fin du II $^{\mathrm{e}}$ siècle ${ }^{53}$. Dans ces conditions, l'inscription locale date bien du II $^{\mathrm{e}}$ siècle $^{54}$.

\section{Les personnes}

Silicia Namgidde porte les duo nomina. C'est une citoyenne africaine. Le CIL a supposé que Q. Silicius Silbanus et Messea Ianuaria (CIL X, 3630, Misène) pouvaient être les parents de la défunte. Mais cette hypothèse est incertaine comme le reconnaît le CIL. Le gentilice est implanté en Afrique Proconsulaire et en Numidie ${ }^{55}$. Une forte concentration du gentilice se situe à Thibilis, à l'est de la Numidie (huit exemples ${ }^{56}$ ), l'autre concentration se trouve à Lambèse (sept exemples ${ }^{57}$ ). Les autres références se situent en Lusitanie (surnom Silicianus, CIL II, 205), dans le Latium (trois références, CIL X, 1996; XIV, 2720. Silbanus est dit natione Afer dans CIL X, 3630), en Gaule Narbonnaise (ILN, Fréjus, $24^{58}$ ), en Dalmatie ( $A E 2008,1071$, sous la forme Siliciana, CIL III, 2278). Le cognomen Namgidde/Namgedde,

à Lyon, la qualification des défunts demeure rare jusqu'en 70 apr. J.-C., BERTHET, JeanFrançois, PAGNON, Bruno, "Le vocabulaire moral des inscriptions de Lyon et de Vienne ", dans La langue des inscriptions latines de la Gaule, Actes de la table ronde tenue au CERGR les 6 et 7 octobre 1988 (univ. Lyon III), CERGR, 7, 1989, p. 44 (à Lyon), p. 51 (à Vienne); BuRNAND, Yves, "Infelicissimi superstites. L'expression du deuil dans les épitaphes arlésiennes du Haut-Empire ", dans Defosse, Pol (dir.), Hommages à Carl Deroux, III, Histoire et épigraphie, droit, coll. « Latomus; 270 ", 2003, p. 111. Les qualifications dans les AlpesMaritimes se rapprochent aussi du modèle viennois (à partir du milieu du II siècle), KATARZYNSKI, Nicolas, Memorias et titulos fecerunt in Alpibus Maritimis..., op. cit., p. 313. Les références mentionnant les sentiments en Afrique Proconsulaire et en Numidie sont proportionnellement très peu abondantes (335 exemples pour pientissim- ou carissim-, 466 pour piissim- ou merentissim-) au regard de l'immense documentation africaine (près de 40000 inscriptions). Les mentions de gentilices impériaux, Flavius, Aelius, montrent bien une existence de tels sentiments depuis les Flaviens au moins avec prudence.

53. LE GLAY, Marcel, "Les critères onomastiques de datation ", dans Inscriptions latines de Gaule Narbonnaise, Actes de la table ronde de Nîmes, 25-26 mai 1987, Publication des Actes, no 20, 1989, p. 14.

54. L'inscription est ordinairement datée du $\mathrm{II}^{\mathrm{e}}$ siècle par la plupart des historiens, tel LANGOUËT, Loïc, op. cit., p. 31. Mais il n'exclut pas le premier siècle de notre ère. N. MATHIEU donne les tria nomina et le formulaire du texte, sans s'étendre davantage dessus, pour dater l'épitaphe du II ${ }^{\mathrm{e}}$ siècle, MathiEu, Nicolas, "Corseul : Lever de rideau sur une capitale? ", art. cit., p. 21. La fin du Ir siècle est aussi proposée par SEYMOUR DE RICCI, Robert, " Répertoire épigraphique de la Bretagne occidentale ", art. cit., p. 263. Quant à G. GuENNOU, il mentionne la formule $H S E$ et l'usage de $D M$ pour dater la pierre de la fin du premier ou du second siècle, cf. du même auteur, GuENnou, Guy, La cité des Coriosolites, op. cit., p. 84. Il s'était appuyé sur HAтT, Jean-Jacques, La tombe gallo-romaine, Picard, réed. 1986, p. 18-19.

55. Dix occurrences en Afrique Proconsulaire; neuf en Numidie.

56. CIL VIII, 5621, 19050; GselL, Stéphane, Inscriptions latines de l'Algérie, Paris, 1922 = désormais abrégé ILAlg., II/2, 5841, 5842, 5843, 5844, 5845, 5847.

57. CIL VIII, 2723 = VIII, 18120, 4065, 4066, 18067, 18068, 18328, 18457. En ajoutant les cognomina, les occurrences CIL VIII, $3928=18438,4064$ (Silicianus), le total du nom se porte à neuf.

58. L'autre occurrence, à Glanum, est plus hypothétique : Si[l][ic]ia[nus (?)], CAG-13/2, p. 394 . 
essentiellement féminin ${ }^{59}$, se concentre en Afrique Proconsulaire avec tout de même deux exemples en Maurétanie Césarienne (CIL VIII, 9199 et p. $1970 ; 20556$ ) et un unicum en Numidie (CIL VIII, 17659). Il est cependant utilisé comme gentilice, particulièrement à Thubursicu Numidarum où apparaissent six exemples ${ }^{60}$. Il n'est pas étonnant de le retrouver dans cette province, le cognomen étant probablement punique dont la racine nam-signifierait "le chanceux ${ }^{61}$ ". Nous remarquons que mis à part Dougga, Masakin, Thala, Mactar et Thigibba, le gentilice et le cognomen se rencontrent surtout dans une zone rassemblant Thibilis et Thubursicu Numidarum, des lieux relativement proches, où sont justement attestées des personnes portant le gentilice Silicius. Il n'est pas impossible que la défunte soit originaire de cette région mais nous manquons de moyens pour le prouver. Silicia Namgidde est donc, comme en témoigne son onomastique, une Africaine.

Son fils, Ianuarius, porte des tria nomina. Son gentilice, Flavius, est romain. Il n'a pas le prénom des empereurs flaviens, Titus. Il n'a donc pas reçu le droit de cité de ces empereurs. Il descend sans doute d'ancêtres ayant reçu le droit de cité sous les Flaviens ou ayant été promus par des personnes portant le gentilice Flavius. L'épitaphe n'autorise pas à dire que Flavius Ianuarius était durablement installé à Corseul ${ }^{62}$ mais elle ne dit pas non plus qu'il n'était que de passage. Contrairement à l'Afrique du Nord, le gentilice du dédicant est très peu représenté en Lyonnaise (dont la majorité habite Lyon) ou en Aquitaine ${ }^{63}$. À Rennes, nous trouvons le gentilice abrégé de Postuminus, un notable riédon qui a reçu des statues en son honneur dans la basilique du temple de Mars Mullo ${ }^{64}$. Le cognomen du dédicant,

59. L'un se retrouve sur une inscription honorifique de Masakin datée d'entre l'automne 106 et l'automne 107 (mention de la XI ${ }^{\mathrm{e}}$ puissance tribunicienne de Trajan) où est mentionné Namgiddo ( $A E$ 1938, 43). L'autre cas, un dénommé Namgiddo Casca est mentionné à Sétif, MASSIERA, Paul, "Inscriptions de la région de Sétif ", Bulletin archéologique du comité des travaux historiques et scientifiques, années 1951-1952, Paris, 1954, p. 240. L'anthroponyme est utilisé comme gentilice. C'est probablement une inversion cognomen-gentilice. Un autre cas du gentilice Casca est effectivement connu à Pompéi ( $A E 1930,123=2001,800)$.

60. Namgidde : ILAlg., I, 1417, 1783 et 1784. Namgedde : CIL VIII, 4906, 4907; ILAlg., I, 1703.

61. Khanoussi, Mustapha, Maurin, Louis, Dougga fragments d'histoire. Choix d'inscriptions latines éditées, traduites et commentées (I $I^{\text {er }} I^{\mathrm{e}}$ siècle), Bordeaux/Tunis, Ausonius Publications, coll. " Mémoires-3 ", 2000, désormais abrégé Dougga, inscr. n 146.

62. Mathieu, Nicolas, "Corseul : Lever de rideau sur une capitale? ", art. cit., 2003, p. 21.

63. En Aquitaine, gentilice non abrégé : $A E$ 1945, 17; CIL XIII, 83, 733, 1294, 1445, 1477, 2418 et p. $706=1353$. Gentilice abrégé : CIL XIII, 617, 990, 1098, 1124 10032.01, 11108; $C A G / 03$, p. 155. En Lyonnaise, gentilice non abrégé : CIL XIII, 1856, 1857, 1858, 1950, 1995, 2026, 2139, 2140, 2143, 2933, 3056, 3090, 11204; $A E 2009,866$; $C A G / 76-02$, p. 223 = p. 225. Gentilice abrégé : CIL XIII, 1745, 1752, 1753, 1754 (même personne que sur le 1752 et 1753), 1804, 1839, 1969, 2004, 2103, 2141, 2142, 2605, 2862, 2993; AE 1976, 428, 443; ILTG, 234; AE 1969/70, 405a, 405b et 405c (même personne).

64. Chastagnol, André, "L'organisation du culte impérial dans la cité à la lumière des inscriptions de Rennes ", dans RouANET-LIESENFELT, Anne-Marie, La civilisation des Riedones, Brest, Archéologie en Bretagne, 2e supplément, 1980, p. 190; BouSQUET, Jean, « Inscriptions de Rennes ", art. cit., p. 109-122. 
Ianuarius, pourrait être un nom punique traduit en latin, signifiant « fils de (= né à) la pleine lune ${ }^{65}$ ». Il est particulièrement courant en Afrique du Nord (plus de 1000 exemples $^{66}$ ).

Nous ne pouvons pas déterminer si Silicia Namgidde a bien accompagné son fils durant son voyage d'Afrique en Armorique ou si elle l'a rejoint à Corseul. Il est vain de proposer leur itinéraire depuis l'Afrique sur route ou par la mer. Burdigala (Bordeaux) est l'un des points de passage maritime possibles vers l'Armorique. À Burdigala, un texte témoigne de la présence d'un Coriosolite, Reginianus, qui a élevé une tombe à son épouse décédée Donata ${ }^{67}$. Les mentions d'Africains ne semblent pas être considérables en Gaule. Un Carthaginois, de natione Afer, est cité à Lugdunum (CIL XIII, $2000)^{68}$. J.-M. Lassère avance cinq cas à Lyon, Trêves, Troyes, Dijon et Langres sur la base de l'anthroponyme Mascel ${ }^{69}$. Des allusions africaines sur des surnoms comme Africanus, à Auxerre (CIL XIII, 2921) et à Vienne (ILN, Vienne, I, 226) ou Afer à Seyssuel (Vienna, ILN, Vienne, I, 1841) doivent être prises avec prudence. Des témoignages des produits de céramique montrent l'existence de circuits économiques entre l'Afrique et l'Armorique bien qu'ils soient peu considérables ${ }^{70}$.

\section{Le formulaire}

La formule peut se décliner de deux manières selon que sacrum apparaît (DMS, " consacré aux Dieux Mânes ") ou non (DM, "Aux Dieux Mânes "). L'invocation aux Dieux Mânes apparaît aussi sur un autre monument à SaintIgneuc sous la forme $D M^{71}$. Le sigle $D M S$ n'est pas très répandu en Gaule : à peine quatre exemples en Lyonnaise ${ }^{72}$, trois en Aquitaine ${ }^{73}$. L'invocation Dis Manibus Sacrum est particulièrement fréquente en Afrique Proconsulaire et en Numidie (plus de 12000 exemplaires). L'Afrique Proconsulaire compte près de 8000 cas, la Numidie 4000. Dans cette province où se situe une forte concentration des porteurs du gentilice Silicius, on trouve environ 430 cas à Thibilis et près de 960 cas à Lambèse. À Thubursicu Numidarum (Afrique

65. LASSERE, Jean-Marie, Vbique populus. Peuplement et mouvements de population dans l'Afrique romaine de la chute de Carthage à la fin de la dynastie des Sévères (146 a. C.-235 p. C.), Paris, CNRS, 1977, p. 451, 453.

66. KaJANTO, Liro, The Latin Cognomina, Helsinki, 1965, p. 218.

67. CIL XIII, 616; MAURIN, Louis, NAVARRO CABALlERO, Milagros, Inscriptions Latines d'Aquitaine (ILA), Bordeaux, De Boccard, 2010, inscription n ${ }^{\circ} 109$.

68. Sur les émigrants africains, voir LASSERE, Jean-Marie, Vbique populus..., op. cit., p. 626633. Un autre Carthaginois se trouve à Iuliobona $(A E 1978,500)$ mais la restitution $c$ (iuis) K(arthaginensis) n'est peut-être pas assurée.

69. CIL XIII, 2140, 2269, 5337, 5811, 11092. Ibidem, p. 630.

70. Galliou, Patrick, "Afrique et Armorique. À propos d'une amphore d'Afrique du Nord mise à jour à Rennes ", Antiquités Africaines, no 26, 1990, p. 223-229. L'article cite Silicia Namgidde pour montrer l'existence du contact entre l'Afrique et l'Armorique.

71. $C A G / 22$, p. 169 .

72. $A E 2009,876 ; C A G / 69$, p. $716=A E 1976,432$; CIL XIII, 2956, 3007.

73. CIL XIII, 266, 1304; $C A G / 12$, p. 402. 
Proconsulaire), nous recensons approximativement 380 exemples. L'emploi du nominatif après $D M$ est très majoritaire à Carthage comme à Lambèse ${ }^{74}$. Fait remarquable, presque toutes les inscriptions de Thubursicu Numidarum adoptent la séquence $D M S$ + nominatif ${ }^{75}$ comme à Thibilis ${ }^{76}$. À Nantes, une seule inscription sur une quinzaine évoque le nominatif (CIL XIII, 3213) ${ }^{77}$. Si on ne dispose évidemment pas de comparaison possible avec le matériel de Corseul, l'unique occurrence du nominatif à Nantes et son emploi récurrent en Afrique suggèrent que l'emploi $D M S$ + nominatif pourrait être dû au dédicant plutôt qu'au lapicide, même s'il convient d'être prudent sur ce point.

Eximia est un mot rarement employé dans les inscriptions. Il se rencontre 140 fois environ dans l'épigraphie du monde romain, la majorité se situant à Rome, dans le Latium (près de vingt-six cas) et en... Afrique Proconsulaire (une cinquantaine de cas) ${ }^{78}$. L'association eximia pietate dans les épitaphes est exceptionnelle. On la retrouve en Vénétie-et-Histrie (pietatis eximiae, CIL V, 4029 et p. 1078), à Rome (eximiae pietatis, CIL VI, 10770), en Afrique même (eximiae pietatis, CIL VIII, 23933; ILAlg., I, 1556; eximiam pietatem, CIL VIII, 1419 = VIII, 15212), en Germanie Supérieure (eximiae pietatis, CIL XIII, 6968). Parmi ces inscriptions, une seule l'attribue à une mère (CIL XIII, 6988). Une autre variante existe toutefois. C'est le cas d'ob eximiam in se pietatem eius (Vénétie-et-Histrie, CIL V, 3417), pietatem eius in se eximiam (même région, CIL V, 4354), ob eximiam eius erga se pietatem (Rome, CIL VI, 32414 et p. 3826), ob eximiam erga se pietatem (Ombrie, CIL XI, 5227). Remarquons qu'un seul cas de l'association (ILAlg., I, 1556) est attesté à Thubursicu Numidarum où sont recensés plusieurs Namgidde. Compte tenu du faible nombre de cas proportionnellement à l'échelle du monde romain et aux cas remarquables en Afrique Proconsulaire, l'association est très intéressante à relever sur la stèle armoricaine.

Le mot secuta, utilisé dans le contexte de l'inscription de Corseul (une personne suit une autre), n'est pas fréquemment attesté dans l'épigraphie impériale. On ne le retrouve que trois fois. Les autres références se situent principalement à l'époque chrétienne. Il est remarquable de retrouver les exemples en Afrique (Proconsulaire et Numidie). Deux se situent à

74. PIETRI, Charles, "Inscriptions funéraires latines ", art. cit., p. 1413. Dans les régions militaires d'Afrique, l'emploi du nominatif est banal même si quelques cas de génitif et du datif subsistent, LASSERE, Jean-Marie, "Sur la chronologie des épitaphes des régions militaires ", Bulletin d'Archéologie Algérienne, nº 5, 1971-1974, p. 158. À Lambèse, voir p. 161.

75. Les autres exceptions sont employées au datif : CIL VIII, 4911, 4984, 17166; ILAlg., I, 1537, 4006.

76. Autres exemples au datif ou génitif : CIL VIII, 10852, 18934, 19042, 19057, 19068; ILAlg., II/2, 5048, 5131, 5143, 5640, 5658 .

77. La majorité des cités de Lyonnaise adopte la séquence $D M+$ génitif ou $D M+$ datif. Le nominatif est plus rare (CIL XIII, 2533, 2847, 3040, 3235, 3236). Certains cas de nominatif ne sont pas pris en compte parce qu'ils comportent la mention uiuus/a, ainsi CIL XIII, 2602 ou 3240.

78. Le plus proche exemple se trouve à Saint-Denis (ILTG, 334) mais semble être tardif d'après l'emploi de c(larissimae) f(eminae). 
Carthage ${ }^{79}$, l'autre à Constantine ${ }^{80}$. Les inscriptions carthaginoises sont sans équivoque : les défuntes ont suivi leurs maris ${ }^{81}$.

Hic situs/a est n'est pas un phénomène courant en Lyonnaise. La province en compte quatre exemplaires ${ }^{82}$. Le nombre est un peu plus élevé en Aquitaine (14 occurrences $)^{83}$. La rareté est sans doute liée à l'absence de troupes stationnées en Gaule ${ }^{84}$. L'Afrique Proconsulaire et la Numidie sont de grosses pourvoyeuses de la formule (plus de 10000 inscriptions dont 881 à Thibilis, 419 à Thubursicu Numidarum, 119 à Lambèse). L'emploi de la formule dans notre inscription est problématique. Elle est en effet gravée sur les épitaphes essentiellement au $\mathrm{I}^{\mathrm{er}}$ siècle apr. J.-C. Son emploi disparaît passé ce siècle, même s'il subsiste sur de rares monuments comme celui de Lyon (CIL XIII, 2104). Sur cette épitaphe, il y a une association DM et $m$ (emoriae) + texte métrique. Cette formule est tardive. Dans la grande partie de la Gaule, HSE est toujours datable du premier siècle de notre ère ${ }^{85}$. Ici, la formule est pourtant associée à la formule introductive DMS et au gentilice impérial Flauius qui apparaissent à la fin du $\mathrm{I}^{\mathrm{er}}-\mathrm{II}^{\mathrm{e}}$ siècle. L'inscription locale sortirait donc du lot. Le problème se pose autrement si on prend en considération l'équivalent africain. La formule continue à être utilisée pendant les trois premiers siècles ${ }^{86}$. Les gentilices impériaux Ulpius ${ }^{87}$, Aelius ${ }^{88}$ ou Septimius ${ }^{89}$ sont plusieurs fois mentionnés sur des épitaphes. Certains affranchis impériaux d'Hadrien (P. Aelius Chresinus, CIL VIII, $5275=17461$; Felix, CIL VIII, 12667; Primus, CIL VIII, 12915; Optatus, CIL VIII, 24702), et

79. CIL VIII, 12657, 24971.

80. CIL VIII, 7517.

81. CIL VIII, 12657 : Dis Manibus sacrum/Claudiae Ti(beri) f(iliae) Euresis pia uix(it)/ ann(os) XVII Valentinus ex/numero cubiculariorum Aug(usti)/fecit uxori carissimae et ob/ meritis quod se secuta esset/in prouincia Africa h(ic) s(ita) e(st). CIL VIII, 24971 : D(is) M(anibus) s(acrum)/Publiciae Rhodope/nat(ione) Italic(a) uix(it) ann(os) XXII/quae secuta est Publi[cium]/ marit(um) et collib[ertum]/ fecit Publi[cius].

82. Châlon-sur-Saône (CIL XIII, 2613, 2615), Paris (CIL XIII, 3042) et Lyon (CIL XIII, 2104). 83. CIL XIII, 53, 55, 128, 414, 922, 923, 924, 1046, 1121, 1159, 1200, 11008; CAG/65, p. 174. La plus proche attestation près de la Bretagne est Antigny (CIL XIII, 1163). Les exemples sont plus élevés dans le sud de l'Aquitaine.

84. HATT, Jean-Jacques, La tombe gallo-romaine, op. cit., p. 18; KATARZYNSKI, Nicolas, Memorias et titulos fecerunt in Alpibus Maritimis..., op. cit., p. 230-231.

85. HATT, Jean-Jacques, La tombe gallo-romaine, op. cit., p. 18-19. B. Marion a proposé d'élargir la datation jusqu'à la fin du II $^{\mathrm{e}}$ siècle pour la Belgique mosellane. Sa proposition est rejetée par RAPSAET-CHARLIER, Marie-Thérèse, "Hic situs est ou Dis Manibus. Du bon usage de la prudence dans la datation des épitaphes gallo-romaines ", L'Antiquité Classique, $\mathrm{n}^{\circ} 71,2002$, p. 221-227, avec de bons arguments.

86. Cf. LASSERE, Jean-Marie, "Sur la chronologie des épitaphes des régions militaires ", art. cit., 1971-1974 et 1973.

87. CIL VIII, 5048, 11407, 12089, 12857, 12858, 23352, 23534, 24802, 28048 en Afrique Proconsulaire. CIL VIII, 2001 (et p. 931), 2048, 2049, 3548, 18359 en Numidie.

88. AE 1973, 619; CIL VIII, 11748, 12636 (et p. 2459), 12665, 12668, 12669, 12887, 12928, 12929, 12932, 12933, 17028, 24681, 24695, etc. en Afrique Proconsulaire. CIL VIII, 2783 et p. 1739,3015 et p. 1740, 3020, 4626 et p. 952, 4802, 7942, etc. en Numidie.

89. Dougga, n ${ }^{\circ} 151$; CIL VIII, 5102, 5459 et p. 1659, en Afrique Proconsulaire. CIL VIII, 5990, 5991, 5992 en Numidie. 
d'Antonin le Pieux (T. Aelius Eutyches, $A E$ 1992, 811; Fortunatus, CIL VIII, 12883 et p. 2459; Libycus, CIL VIII, 13188) apportent une chronologie plus précise. Une inscription mentionne même trois empereurs en même temps, Septime Sévère, Caracalla et Geta (Auggg., CIL VIII, 24689).

La formule funéraire est écrite en toutes lettres : Hic sita est. L'usage est souvent de la réduire aux initiales $H S E$. En revanche, l'autre formule, uixit an(nis) est partiellement abrégée. Est-ce par souci d'ordinatio que la formule $H S E$ a été développée en toutes lettres? L'éventualité de la rareté de la formule dans la région a-t-elle poussé le lapicide à ne pas raccourcir les lettres? Plus d'une centaine d'exemples de la formule non abrégée $H S E$ sont recensés en Proconsulaire et Numidie ce qui constitue un nombre très faible par rapport à dix mille cas environ. En Afrique pourtant, on la voit parfois coexister avec la formule abrégée sur une même inscription ${ }^{90}$. Il faut toutefois remarquer que la formule non abrégée est intégrée dans une inscription métrique. Si la plupart de tels exemples sont datés du ${ }^{\text {er }}$ siècle apr. J.-C. ${ }^{91}$, un texte mentionne le gentilice impérial Aelius d'un affranchi (CIL VIII, 3323 et p. 1741). Cet exemple montre que la formule non abrégée n'est pas toujours un signe d'ancienneté ${ }^{92}$. Aux questions posées plus haut, nous sommes une nouvelle fois de plus dans l'incapacité de répondre.

Namgidde a " officiellement " un âge de soixante-cinq ans, donné par son fils. La précision de l'âge pour le défunt n'est pas rare ${ }^{93}$. Elle est peutêtre arrondie du fait de l'attraction des multiples de 5 et de $10^{94}$ ou à la mauvaise connaissance de l'état civil personnel. Cependant, il est plutôt vraisemblable qu'elle ait atteint plus de 60 ans. Cet âge marque le début de la vieillesse selon la conception antique ${ }^{95}$. Toutefois, pour les femmes, elle pourrait commencer à partir de 50 ans $^{96}$. En Afrique, l'espérance de vie est de 48 ans pour tous les sexes. Elle est supérieure à celle de l'Espagne dont la moyenne est de 37 ans. La longévité africaine est supérieure de 10 ans à celle de l'Italie rurale et de 15 ans à celle de Rome ${ }^{97}$. La mention d'âge apparaît également sur une autre inscription locale trouvée au Clos

90. CIL VIII, 2211 (une fois pour chaque formule), 28082 (deux fois HSE, une fois hic situs est), 26670 (une fois pour chaque formule), $A E 2006,1774$ (même cas que précédemment).

91. CIL VIII, 990, 2257, 2812 et p. $954=18143,5075,5936,10976,11288,16345,22958$, 23853, 24755, 24862, 28036; $A E 1980,914$ = 1983, 969 .

92. Autres exemples de datation des II $^{\mathrm{e}}$-III ${ }^{\mathrm{e}}$ siècles : CIL VIII, 2401 et p. 1693 (gentilice Aelius), 2817 (gentilice Aurelius, duo nomina deuxième manière), MERLIN, Alfred, Inscriptions latines de la Tunisie, Paris, 1944, no 806 (duo nomina deuxième manière). Sans compter les nombreux cas de Dis Manibus de datation souvent postérieure à la fin du $\mathrm{I}^{\mathrm{er}}$ siècle.

93. LASSERE, Jean-Marie, Vbique populus..., op. cit., 1977, p. 469-470.

94. SALMON, Pierre, "Les insuffisances du matériel épigraphique sur la mortalité dans l'Antiquité romaine ", dans HINARD, François (dir.), La mort, les morts et l'au-delà dans le monde romain, 1987, p. 109.

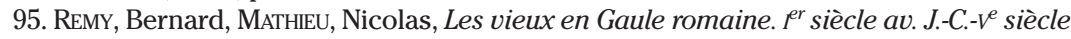
apr. J.-C., Arles, Errance, 2015, p. 11.

96. Ibidem, p. 11 et $164, \mathrm{n}^{\circ} 44$.

97. Corbier, Paul, GriesheIMER, Marc, L'Afrique romaine. 146 av. J.-C.-439 apr. J.-C., Ellipses, 2005, p. 295-296. 
Julio, bien qu'incomplète ${ }^{98}$. La formule est restituée sous la forme [q(ui) uixit an]n(is) $C X$ ou [uixit an]n(is) $C X$. La présence de cette mention d'âge à Corseul expliquerait la version partiellement abrégée de la formule sur la stèle puisqu'elle y est suffisamment connue. D'autres occurrences apparaissent à Nantes (22 ans dans CIL XIII, 3115 et 16 ans dans XIII, 3121). Plus loin, en Normandie, signalons $A E$ 1990, 721 (dédiée à la Memoria) à Caen, trois références à Lisieux $(C A G / 14$, p. 102; CIL XIII, 3179, 3180, datables du $\mathrm{I}^{\mathrm{er}}$ siècle de notre ère d'après l'usage du nominatif et l'absence de $D M$ ).

Mais il existe une spécificité africaine pourtant non mentionnée sur l'inscription locale : pius/a uixit annis. Extrêmement courante (près de 5000 occurrences), la formule est le plus souvent réduite aux initiales $P($ ius $/ a)$ $V$ (ixit) A(nnis) ${ }^{99}$. Un Africain peut utiliser indifféremment pius/ia uixit annis ou uixit annis. En effet, l'épithète pius accompagne toujours l'âge en Afrique Proconsulaire mais manque le plus souvent en Numidie ${ }^{100}$. Ainsi, on trouve plus de 340 cas à Thubursicu Numidarum alors que Lambèse en compte à peine 14. Le nombre des inscriptions sans l'occurrence pius/pia reste très élevé dans les deux provinces (près de 10000). Suivant ces cas, si lanuarius est bien l'auteur du texte, il a choisi la formule uixit annis plutôt que pia uixit annis.

Les formules DMS et HSE sont des poncifs de l'épigraphie africaine. Certes, la documentation épigraphique du nord de la Gaule Lyonnaise présente un nombre faible par rapport à Lugudunum ${ }^{101}$. Si ce nombre est faible, alors les formules devaient être peu nombreuses. Pourtant, ce n'est pas parce que le nombre est faible que les occurrences du formulaire le sont aussi. Le corpus nantais compte 47 inscriptions ${ }^{102}$. Il ne compte pourtant aucun cas avec DMS. Cet exemple nous fait douter de l'hypothèse de l'origine locale de la formule. En parlant de $D M S$, l'Afrique suit particulièrement la séquence $D M+$ nominatif (comme sur notre épitaphe) alors que la Gaule Lyonnaise préfère le datif ou le génitif. Les mentions eximia pietate et filium secuta ne sont pas des stéréotypes de l'épigraphie mais une expression spontanée du dédicant. Ces mots sont surtout signalés en Afrique. Même la paléographie renforce la piste africaine (grandes lettres pour la défunte, petites lettres pour le dédicant) ${ }^{103}$. Le texte peut être préparé par un ordi-

98. $I L T G, 340=C A G / 22$, p. 121 .

99. L'association du mot pietas avec la formule est peu utilisée dans l'épigraphie d'Afrique et de Numidie. Le mot est mentionné six fois dont trois sur des textes métriques, CIL VIII, 12159 (métrique) 12626, 14740,15569 et p. 2697 (métrique), 24807; ILAlg., I, 1362 (métrique, à Thubursicu Numidarum).

100. LASSERE, Jean-Marie, "Recherche sur la chronologie des épitaphes païennes de l'Africa ", art. cit., 1973, p. 21.

101. En consultant le CIL XIII, l'ensemble des inscriptions de Lyon compte 719 occurrences. Les autres cités de la Lyonnaise rassemblent 806 inscriptions, ce qui constitue un nombre proportionnellement faible par rapport à Lyon.

102. MATHIEU, Nicolas, " L'épigraphie dans l'Ouest armoricain... », art. cit., 2011, p. 135-137. 103. La paléographie peut être une stratégie pour attirer ou repousser le passant. Le mausolée d'Auguste portait de longs textes gravés sur des plaques de bronze dont les lettres plus petites exigeaient du passant qui voulait en prendre connaissance plus d'attention qu'une simple épitaphe, CORBIER, Mireille, Donner à voir, donner à lire, mémoire et com- 
nator ${ }^{104}$ ou un lapicide et être proposé au client ${ }^{105}$. Sinon, c'est le client qui se charge de faire graver une inscription à son goût soit par testament ${ }^{106}$, soit de son vivant pour lui ou ses proches. Le texte établi conformément aux volontés du dédicant étranger n'est pas nouveau en Armorique. Une dédicace religieuse à Neptune, provenant de Douarnenez (Finistère), porte la forme classique de l'inscription suggérant une personne étrangère à la région ${ }^{107}$, ce qui est confirmé par l'origine sans doute narbonnaise du dédicant. En tenant compte des coûts liés aux déplacements et tout ce qu'il implique (hébergement et restauration), il n'est pas improbable de voir en Ianuarius un personnage avec des revenus élevés. Sachant que la gravure est un acte coûteux ${ }^{108}$, il peut se permettre de rédiger plusieurs lignes du texte et de les passer à un ordinator ou à un lapicide, surtout lorsqu'il veut honorer efficacement la mémoire de sa mère.

Les indices conduisant à l'Afrique étant variés, l'inscription est bien plus africaine qu'armoricaine. L'intérêt de l'inscription ne réside pas uniquement dans l'évocation des étrangers à Corseul, avec une expression peu employée eximia pietate filium secuta, mais rend compte de l'importation des habitudes épigraphiques depuis l'étranger. Le monument est tout aussi remarquable à cause du décor dépouillé très peu connu en Bretagne. Pour toutes ces raisons, l'inscription est bien digne du classement comme monument historique 1911.

munication dans la Rome ancienne, CNRS Éditions, Paris, 2006, p. 23. À l'inverse, Caligula, pressé par les habitants de Rome de rendre public le règlement des impôts, les faisait afficher en lettres très petites (Suétone, Caligula, XLI), CORBIER, Mireille, op. cit., p. 44.

104. Un ordinator est une personne qui compose et ordonne le texte sur la pierre d'après une rédaction établie auparavant sur un papyrus ou une tablette, MALLon, Jean, "L'ordinator des inscriptions ", dans : Comptes rendus de l'Académie des Inscriptions et Belles-Lettres, 99/1, 1955, p. 129. Il mentionne l'exemple de l'inscription bilingue à Palerme par ces mots Tituli h[e]ic ordinantur et sculpuntur (CIL X, 7296).

105. L'un des meilleurs exemples de textes prêts à l'emploi est une étourderie du graveur distrait qui a recopié le texte en oubliant de supprimer la mention hic corpus iacet pueri nominandi soit " ci-git le corps de l'enfant à nommer " ( $A E$ 1931, 112 à Annaba). Un même atelier peut reproduire un même texte (sauf les noms restant à remplir) sur plusieurs pierres. Ainsi, sur la côte monégasque, la paléographie strictement similaire et la formule récurrente pientissim(-) parentes infelicissimi apparaissent sur le CIL V, 7826 et LAGUERRE, Georgette, Fouilles de Cemenelum II, Inscriptions antiques de Nice-Cimiez (Cemenelum, Ager Cemenelensis), E. de Boccard, Paris, 1975, n 125; KATARZYNSKI, Nicolas, Memorias et titulos fecerunt in Alpibus Maritimis..., op. cit., p. 731. Une épitaphe de l'urne cinéraire du Château de Nice est fortement soupçonnée d'être préinscrite sur la face antérieure de la cuve, KATARZYNSKI, Nicolas, GAYET, Frédéric, "L'épigraphie antique du Château de Nice ", dans BourRon, Bouiron, Nice. La colline du Château, histoire millénaire d'une place forte (Mémoires Millénaires), Nice, 2013, p. 66-67. 106. Exemple dans la fiction de Pétrone, Le Satyricon, LXXI. CORBIER, Mireille, op. cit., p. 22. 107. ILTG, 338; SANQuER, René, op. cit., 1973, p. 225; ÉvEILlaRD, Jean-Yves, Le RouX, Patrick, op. cit., 2013, p. 7-16.

108. D’après SALMON, Pierre, "Les insuffisances du matériel épigraphique sur la mortalité dans l'Antiquité romaine ", art. cit., 1987, p. 101-102, la rédaction d'une inscription funéraire coûte cent sesterces au minimum. 


\section{RÉSUMÉ}

Une inscription mentionnant une épitaphe de Silicia Namgidde (originaire de l'Afrique) conservée dans l'église Saint-Pierre en Corseul (Côtes-d'Armor) n'a pas, jusqu'alors, fait l'objet d'une étude approfondie autant du point de vue du monument que du texte. Après analyse, il semble que le monument soit une stèle. L'analyse du formulaire funéraire du $\mathrm{II}^{\mathrm{e}}$ siècle et de l'écriture montrent une pratique épigraphique particulièrement fréquente en Afrique plutôt qu'en Gaule. Le texte a sans doute été proposé par le client, qui est le fils de la défunte, Caius Flavius Ianuarius, en vue d'être gravé sur la pierre par le lapicide.

\section{ABSTRACT}

An inscription mentioning an epitaph of Silicia Namgidde (native to Africa) preserved in the Church of Saint-Pierre in Corseul (Côtes-d'Armor, Brittany) has hitherto not been the subject of much research. Close examination of the monument suggests that it is probably a stele. The analysis of the funeral form the second century and of the writing shows an epigraphic practice especially common in Africa rather than in Gaul. The text was probably proposed by the client, who was the son of the deceased, Caius Flavius Ianuarius, before being engraved on the stone by the stonecutter. 